\title{
Nation und Narration: Eine vergleichende Untersuchung der Beiträge der Brüder Zhou und der Brüder Grimm zur Folklore
}

Als die Brüder Grimm zur Geschichte der Literatur und zu literarischen Motiven aus aller Welt forschten und die Welt in ihren Geschichten, Märchen und Erzählungen $\mathrm{zu}$ verstehen und zu verbinden begannen, schloss der Kaiser von China das Tor zur Welt und schwelgte in der Vorstellung, dass das ,Reich der Mitte“ im Zentrum der Welt liege und fortgeschrittener sei als alle anderen Länder, weshalb die Kontakte mit diesen unnötig seien. Ein Jahrhundert später diskutierten die Brüder Zhou im Zusammenhang mit dem Chinesisch-Japanischen Krieg die Begriffe Kind, Nation, Nationalität, Märchen und Mythen, Themenbereiche, mit denen sich die Brüder Grimm im 19. Jahrhundert im Kontext der Napoleonischen Kriege beschäftigten. Warum haben sich die beiden Brüderpaare Grimm und Zhou gerade vor der Geburtsstunde einer erhofften modernen Nation, obwohl aus verschiedenen Epochen und so unterschiedlichen Nationen stammend, mit den gleichen Gedanken beschäftigt? Ist dies ein Zufall oder verbirgt sich dahinter eine konkrete Ursache? Der vorliegende Beitrag widmet sich diesen bisher kaum beachteten Fragen.

Knapp ein Jahrhundert, nachdem die Brüder Jacob Grimm (1785-1863) und Wilhelm Grimm (1786-1859) ihre Gedanken zu Poesie und Nation zu Papier gebracht und sich mit Begriffen wie Märchen, Volk, Kind sowie der Beziehung zwischen Märchen und Erziehung bzw. Bildung beschäftigt hatten, sind zwei chinesische Brüder - Zhou Shuren (bekannt unter dem Künstlernamen Lu Xun, 1881-1936, der bedeutendste Schriftsteller der chinesischen Moderne; vgl. Kubin 1985, 9) und Zhou Zuoren (1885-1967) -, die als Vertreter der Avantgarde eine bedeutende Rolle bei der Gründung und Verbreitung der chinesischen modernen Literatur und Kinderliteratur spielten, auf die gleichen Gedanken gekommen, obwohl sie vor einem anderen historischen Hintergrund wirkten.

Im Jahre 1857, als die Kinder- und Hausmärchen in der Ausgabe letzter Hand erschienen, befand sich China mitten im Zweiten Opiumkrieg, der die

\footnotetext{
Note: Dieser Beitrag repräsentiert ein Segment der Vorarbeiten zu einem laufenden Forschungsprojekt (Nr. 19BWW059, unterstützt von The National Social Science Fund of China) mit dem Arbeitstitel: Die mythische Wurzel der Grimmschen Märchen und die deutsche Nationalität.
}

Ә Open Access. (C) 2021 Wang Liping, publiziert von De Gruyter. (c) BY-NC-ND Dieses Werk ist lizensiert unter einer Creative Commons Namensnennung - Nicht-kommerziell - Keine Bearbeitung 4.0 International Lizenz. https://doi.org/10.1515/9783110682427-007 
Vorstellung, dass China als das ,Reich der Mitte“ im Zentrum der Welt liege und fortschrittlicher als alle anderen Länder sei, erschütterte.

Durch die Niederlage in den zwei Opiumkriegen wurde die chinazentrische Weltanschauung der Chinesen aus den Angeln gehoben. Das tausendjährige autarke Wirtschaftssystem geriet in eine fundamentale Krise, und die geschlossenen Grenzen des Kaiserreichs wurden durch die ungleichen Verträge mit den Engländern und Franzosen gezwungenermaßen geöffnet. Das chinesische Volk wurde sich jetzt seines halbfeudalen und halbkolonialen Zustands bewusst und hatte sich der Frage zu stellen, wie die nationale Krise zu überwinden sei.

In den drei Dezennien von 1861 bis 1894 erhob sich die Yangwu-Bewegung zur Modernisierung und Selbststärkung des Landes (vgl. Leutner 1990, 55). In der Hoffnung, durch die Einführung moderner westlicher Technik in der Militär- und Zivilwirtschaft den Rückstand des Landes zu beseitigen, fingen die Reformer an, vom Westen zu lernen, um ihn zu besiegen. Neue Schiffe, Fabriken und Schulen werden gebaut, fremde Fächer und Sprachen werden gelehrt. Studenten werden ins Ausland entsandt. Darunter waren die Brüder Zhou, die wegen der erfolgreichen Meiji-Reform als Studenten nach Japan gesandt worden waren. Während dieser Aufenthalte sind die Brüder auf die westliche Literatur gestoßen und haben jeweils auf ihre ursprünglichen Pläne, Medizin bzw. Architektur zu studieren, verzichtet. Literarisches Schaffen und Übersetzungen aus dem Japanischen, Deutschen und Englischen sowie von griechischen Mythen, von Märchen, Novellen etc. samt Literaturkritik und -theorien machten die Brüder zu Begründern der modernen chinesischen Literatur bzw. der Kinderliteratur. Im Vergleich dazu waren die Brüder Grimm die Mitbegründer des Fachs der Germanistik. Zwar war Lu Xun nicht Bibliothekar wie die Brüder Grimm $^{1}$, aber er setzte sich für die Gründung mehrerer Bibliotheken ein. Zhou Zuoren arbeitete für die Bibliothek der Peking Universität; er ist später Direktor dieser Bibliothek geworden. Beide Brüder lehrten Literatur an der Peking Universität, wie die Brüder Grimm an der Berliner Friedrich-Wilhelms-Universität (der heutigen Humboldt-Universität). Beide Brüderpaare waren wegen des gesellschaftlichen Abstiegs ihrer Familien im jungen Alter von ungefähr zehn Jahren mit der Herausforderung eines sie lebensweltlich umgebenden finanziellen und sozialen Gefälles konfrontiert. Dank der Unterstützung durch Verwandte konnten sie ihren Bildungsgang fortsetzen und entdeckten beim Studium sol-

1 Jacob Grimm war 1808 „Bibliothekar in der Privatbibliothek des westfälischen Königs“ sowie „1816 zweiter Bibliothekar an der Kasseler Bibliothek im Museum Fridericianum“ (Schede 2004, 182). Wilhelm Grimm war „1814 Bibliothekssekretär an der Kasseler Bibliothek im Museum Fridericianum“ sowie „1830 Bibliothekar an der Universitätsbibliothek“ (Schede 2004, 184). 
cher Fächer, die zunächst mit Literatur nichts oder wenig zu tun hatten, die Leidenschaft für die heimische und ausländische Literatur. Im Vergleich zum jeweiligen älteren Bruder sind sowohl Wilhelm Grimm als auch Zhou Zuoren sanfter, poetischer, so dass ihre Werke sich als zeitgemäß und zeitlos bewähren. Für diese Begabung lieferte Jacob Grimm in der Rede auf Wilhelm Grimm einen Grund:

Man hört wol sagen, dasz in gesegneter ehe die älteren kinder mehr dem vater, die jüngeren mehr der mutter nachschlagen, sowie dasz unter den söhnen der erste minderbegabt sei als der zweite, diesen aber der dritte übertreffe, wie auch in kindermärchen der dritte hervorgehoben wird [. . .].

(Grimm 1864, 165)

Wilhelm Grimm stand als der jüngere der beiden in der Forschung eher im Schatten des Bruders, um mit Lothar Bluhm $(2006,148) \mathrm{zu}$ sprechen. Ähnlich zurückhaltend ist die Forschung im Westen zu Zhou Zuoren, der ebenfalls im Schatten seines großen Bruders steht. In China fand die Forschung zu seinem Leben und Werk wegen seiner Kollaboration mit der von Japan dirigierten Marionettenregierung ${ }^{2}$ bis in die 1980er Jahre praktisch nicht statt. In Deutschland arbeitete Jacob Grimm 1808 als Bibliothekar in der Privatbibliothek des westfälischen Königs Jérôme, was aber nie seinem Ruf geschadet hat. Danach hat er das Circular wegen Aufsammlung der Volkspoesie (1815) veröffentlicht, während Zhou Zuoren $^{3}$ schon vor seiner Kollaboration mit Japan sein Zhengqiu Shaoxing ergetonghua qi [Zirkular wegen Aufsammlung der Kinderlieder und Märchen in Shaoxing $]^{4}$ (1914) verbreitet hatte. 1918, 1920 und 1922 hat sich diese Sammlung auf nationaler Ebene um Volkslieder erweitert. Beide Sammler richten sich nach der Prämisse, dass „[a]lles dieses getreu und wahrhaftig ohne Schminke und Zuthat aus dem Mund der Erzählenden gefaßt [. . .] werden [muß]“ (zit. nach Petzoldt 1999, 113-114).

Beide Sammeltätigkeiten waren von demselben Mann inspiriert worden: Johann Gottfried Herder (1744-1803). Die ,Stimme des Volks` wird in dessen Nachfolge gehört und geschätzt. Eine Arbeitsmethode, die auf ,Treue und Wahrhaftigkeit' beruht, pflegen sie beide. Während Jacob Grimm ,Wahrheit

2 Im März 1940 wurde in Nanking eine von Japan unterstützte Marionettenregierung unter der Leitung von Wang Jingwei (1883-1944) eingesetzt, der schon zuvor mit der von Jiang Jieshi geführten Regierung in Chongqing gebrochen hatte und für Japans Interessen arbeitete (vgl. Scherer 2001, 475).

3 Lu Xun hat im Februar 1913 in der Monatsschrift der Editionsstelle des Bildungsministeriums dazu aufgerufen, Volkslieder, Sprichwörter, Legenden und Märchen zu sammeln.

4 Diese und alle folgenden Übersetzungen aus dem Chinesischen wurden von der Verfasserin angefertigt. 
und Geist' der gesammelten Texte so original wie möglich zu bewahren beabsichtigte, damit der ,wahre Geist der deutschen Nation' herauskristallisiert werde, wollte Zhou Zuoren die Volkspoesie, besonders auf dem Lande, sammeln. Sie äußere nämlich die ,unverfälschte Stimme des Volks‘, also des Individuums, statt der geltenden Doktrinen und könne sich gegen die herrschenden alten Moralregeln behaupten, die von konfuzianischen Schriften gepredigt wurden. Diese verzehrten seiner Meinung nach die Menschen und richteten sie gleichsam zu Menschenfressern ab. Da die Brüder Zhou glaubten, dass die Kinder Hoffnungsträger seien, die von der seelenfesselnden und -raubenden Moral- und Wissensinstanz des althergebrachten Bildungssystems durch neue Literatur befreit werden könnten, verbindet sich die Volksliteratur als eine Neue Literatur mit der Kindererrettung, was wiederum die Etablierung der Kinderliteratur begünstigt. Kinder werden dadurch nicht nur entdeckt, sondern auch ikonisiert: „Am Anfang der Menschheitsentwicklung stehend, verkörperten das Kind und der Rekurs auf Ursprung und Natur die Notwendigkeit, den bisherigen Entwicklungsverlauf von Gesellschaft und Zivilisation zu korrigieren“ (Frick 2002, 122).

Nach Wilhelm Grimm bewahren Kinder ebenso das Ursprünglich-Natürliche auf. Aber nicht wie bei Zhou Zuoren im anthropologischen, sondern im religiösen Sinne. Grimm zitiert aus dem Matthäusevangelium und kommentiert:

,Lasset die Kindlein und wehret ihnen nicht zu mir zu kommen, denn solcher ist das Himmelreich'; dieser heilige Spruch bewährt sich durch alle Zeiten, überall geht das Leben des Menschen auf, wie eine Blume, ehe sie die stechende Sonne blässt und der irdische Staub trübt, in reiner, unversehrter Farbe.

(Grimm 1881, 359)

Deshalb geht innerlich durch die Volkspoesie, so schreiben die Brüder Grimm im Vorwort zu den „Kinder- und Haus-Märchen“,

dieselbe Reinheit, um derentwillen uns Kinder so wunderbar und seelig erscheinen; sie haben gleichsam dieselben bläulich-weißen, mackellosen, glänzenden Augen (in die sich die kleinen Kinder selbst so gern greifen [. . .]), die nicht mehr wachsen können, während die andern Glieder noch zart, schwach und zum Dienst der Erde ungeschickt sind.

(Grimm und Grimm 1812, VIII-IX)

Auf den Aspekt des christlichen reinen Kindes geht das kindzentrierte Konzept der Brüder Zhou nicht ein. Sie halten das Kind für kein Symbol des Göttlichen, sondern für ein selbständiges, autonomes Wesen, das vor den systematischen Versklavungsmaßnahmen der Erwachsenen geschützt werden muss. Die Kinder sollten von der herkömmlichen Aufzucht zu „pietätvolle[n] Söhne[n] und Enkel [n]“, ,gehorsame[n] und loyale[n] Bürger[n]“ (zit. nach Frick 2002, 58) zum Erhalt der hierarchischen Gesellschaft befreit werden. Sie sollten den Status des kleinen Erwachsenen ablegen können und eine altersgemäße Erziehung genießen, 
die die Einbildungskraft der Kinder billigt und fördert. Sonst bleiben sie lebenslang willenlose Untertanen, die den Anspruch auf Freiheit aufgeben, weil sie als Kinder wegen ihres Freiheitsdrangs bestraft wurden. Diese systematische Traumatisierung führt zur Pervertierung des sozialen Verhaltens, wie der französische Sinologe Jean-Pierre Diény ${ }^{5}$ hinsichtlich der damaligen Epoche prägnant zusammenfasst: „China behandelt die Kinder wie Erwachsene und die Erwachsenen wie Kinder“ (Diény 1973, 7).

Um diesem Übel einen radikalen Widerspruch entgegenzusetzen, um eine gesunde Persönlichkeit zu bilden, wird eine komplexe Interaktion zwischen dem Westen und China, der Tradition und der Moderne in Gang gesetzt (vgl. Frick 2002, 21). Die heftige Zurückweisung des kulturellen Erbes und seiner Erziehungstradition hatte zur Folge, dass die in China bisher vernachlässigten Literaturgattungen wie Märchen, Sage und Legende, die bei den Kindern eine breite Rezeption und Popularität fanden, positiv bewertet und der Kinderliteratur zugeordnet wurden. So entstand am Anfang des 20. Jahrhunderts in China, begünstigt durch die 4.-Mai-Bewegung und die Neue-Kultur-Bewegung, eine vielfältige Beschäftigung mit der Übersetzung, Sammlung und Erforschung von Märchen.

Die Brüder Zhou gehören zu den ersten Märchenübersetzern bzw. Märchenforschern Chinas. Den Bezug zu den Brüdern Grimm stellte vor allem Zhou Zuoren her. Er hat zwei Märchen (Strohhalm, Kohle und Bohne auf der Reise; Die Rübe) der Brüder Grimm übersetzt und sich in mehreren Artikeln mit Märchen im begrifflichen und thematischen Sinn auseinandergesetzt. Zwischen Mythen, Sagen und Märchen fand er kaum wesentliche Differenzen. Nur seien die ersten religiös, die zweiten historisch, die letzteren literarisch (vgl. Zhou 2014, 25). Ähnlich äußert sich Jacob Grimm im Vorwort zu den Deutschen Sagen: „Das Märchen ist poetischer, die Sage historischer“ (Grimm 1985, 48).

Zhou Zuoren hat im Artikel Die Übersetzungsprobleme des Märchens Grimms Märchen vorgestellt und die chinesische Übersetzung von Märchen als Tonghua (Kindergeschichte) für falsch gehalten. Er hat stattdessen Minjiangushi (Volksgeschichten) vorgeschlagen, die teilweise den Kindern angeboten werden, da für die Brüder Zhou Volksliteratur nicht automatisch Kinderliteratur ist.

Für die Brüder Grimm diente das Sammeln der Volksliteratur nicht zur Befreiung der Kinder, sondern zur Bildung der nationalen Identität. Sogar das

5 Laut Mary Ann Farquhar (1999, 3) ist Jean-Pierre Diénys Le Monde est à Vous. La Chine et les Livres pour Enfants von 1971 die einzige seriöse Studie zur chinesischen Kinderliteratur im Westen. 
Buch Kinder- und Hausmärchen, das die ,Kinder` im Titel aufweist, ist nach Jacob Grimm

gar nicht für Kinder geschrieben, aber es kommt ihnen recht erwünscht und das freut mich sehr, sondern ich hätte nicht mit Lust daran gearbeitet, wenn ich nicht Glaubens wäre, daß es den ernstesten und ältesten Leuten so gut wie mir für Poesie, Mythologie und Geschichte wichtig werden und erscheinen könnte.

(Zit. nach Steig und Grimm 1904, 271)

Noch einen Schritt weiter geht Jacob Grimm, indem er konstatiert, dass er die Frage, „ob man überhaupt für Kinder etwas eigenes einrichten müsse“, verneinen müsse, und zwar mit folgender Begründung:

Was wir an offenbarten und traditionellen Lehren und Vorschriften besitzen, das ertragen Alte wie Junge, und was diese daran nicht begreifen, über das gleitet ihr Gemüt weg, bis daß sie es lernen, wie eigentlich alle wahre Lehre nur die ist, die das schon vorhandene und bekannte entzündet und erleuchtet, nicht aber eine, die Holz und Feuer beide mitbringt.

(Zit. nach Steig und Grimm 1904, 269)

Wilhelm Grimm, der seit 1819 allein die Sammel- und Editionsarbeit der Märchen durchführte, hat aber in seinem Märchen-Vorwort von 1819 die obenstehende Theorie seines Bruders leicht korrigiert: „Dabei haben wir jeden für das Kinderalter nicht passenden Ausdruck in dieser neuen Auflage sorgfältig gelöscht“ (Grimm 2006, 17).

Dieses Märchenbuch ist zwar praktisch ein Erziehungsbuch für Kinder geworden, aber auch ein Beitrag zur Identitätsbildung der stark von französischer Kultur beeinflussten deutschen Nation. Besonders als 1806 die französischen Truppen in Kassel einmarschierten, änderte sich nach Wilhelms Ansicht „alles von Grund aus: fremde Menschen, fremde Sitten, auf der Straße und den Spaziergängen eine fremde, laut geredete Sprache“ (Schede 2004, 31). Obwohl er die französische Sprache schon seit der frühen Kindheit kannte, war sie für Grimms Wahrnehmung im Rahmen der Besatzung durch Napoleons Truppen fremd, denn die Armee gleiche einem „Unglück, das der Himmel schickt, eine ganze Saat zu Boden geschlagen" hat. Und die Volkspoesie ist wie die am Wege stehenden Sträucher, die „auch der einzige Samen für die Zukunft“ (Grimm 2006, 15) sein könnten. Märchen verstehen die Brüder Grimm als eine die Idee der Nation stiftende Instanz, die dem deutschen Volk einen Anhaltspunkt für die kulturelle Einheit verleiht, indem die Vergangenheit in der Form von Mythen in die Gegenwart transkribiert und damit die Zukunft des Volkes gestaltet wird. Das Kind ist hier ein Reinheitssymbol ursprünglicher Ganzheitlichkeit und dient der Einheitsbildung der deutschen Nation im kulturellen Sinne, als die Einheit im politischen Verständnis noch nicht verwirklicht war. 
Für die Brüder Zhou ist der Zusammenhang von Volk, Märchen und Kind durchaus auch mit der Suche nach einer neuen nationalen Identität verbunden, die die alte chinesische Mentalität, geprägt vom Konfuzianismus, aus den Angeln hebt. Dabei kamen die Stimmen des eigenen Volks in China und die der fremden Kultur im Westen zusammen, um so zur Errettung des Landes bzw. des Kindes beizutragen. Die daraus entstandene Blüte der Märchenforschung ist eine gelungene Interaktion des Eigenen und des Fremden. Mithilfe der westlichen Märchen-Philologie wurden die chinesischen Märchen gesammelt und sortiert. Mit dem Fremden das Eigene zu bilden war auch die literarische Praxis der Brüder Grimm. Ihr theoretischer Leitgedanke verlangt zwar die Distanzierung von fremden Sprachen und Kulturen. In der Realität jedoch stießen sie auf die unvermeidbare Vermischung der französischen und der deutschen Narration, da die Textzeugen ihrer Märchen meistens mit französischen Märchen vertraut waren.

Zusammenfassend lässt sich feststellen, dass die beiden Brüderpaare trotz historischer Differenzen ihre Ideen der Bildung bzw. Umbildung der neuen Nation mit einem Konzept der Bildung bzw. Umbildung der neuen Narration verbinden. Das Märchen hat sich im Verbund mit der Folkloreforschung sowohl am Anfang des 20. Jahrhunderts in China als auch am Anfang des 19. Jahrhunderts in Deutschland als zentrales identitätsstiftendes Organ des Volkes erwiesen und zur Bildung des modernen Staats beigetragen. Das gilt sowohl für das sich in der halbfeudalen und halbkolonialen Gesellschaft befindende China wie auch für das aus kleinen und großen Fürstentümern bestehende Deutschland.

\section{Literaturverzeichnis}

Bluhm, Lothar. „J. und W. Grimm“. Deutsche Biographische Enzyklopädie (DBE). 2., überarbeitete und erweiterte Ausgabe. Hrsg. Rudolf Vierhaus. Band 4. München 2006. 147-148.

Diény, Jean-Pierre. Die Welt gehört den Kindern. Das moderne China und seine Kinderbücher. Weinheim und Basel 1973.

Farquhar, Mary Ann. Children's literature in China. From Lun Xun to Mao Zedong. Armonk und New York 1999.

Frick, Heike. „Rettet die Kinder!“. Kinderliteratur und kulturelle Erneuerung in China, 1902-1946. Hamburg 2002.

Grimm, Jacob. „Vorrede zu: Deutsche Sagen. Band 1. 1816“. Jacob Grimm und Wilhelm Grimm: Schriften und Reden. Ausgewählt und herausgegeben von Ludwig Denecke. Stuttgart 1985. 47-55.

Grimm, Jacob. „Rede auf Wilhelm Grimm“ (1860). Kleinere Schriften von Jacob Grimm. Band I. Berlin 1864. 163-187. 
Grimm, Jacob, und Wilhelm Grimm. Kinder- und Hausmärchen. Ausgabe letzter Hand mit den Originalanmerkungen der Brüder Grimm. Mit einem Anhang sämtlicher, nicht in allen Auflagen veröffentlichter Märchen und Herkunftsnachweisen herausgegeben von Heinz Rölleke. Band 1. Stuttgart 2006.

Grimm, Jacob, und Wilhelm Grimm. Kinder- und Hausmärchen. Band 1. Berlin 1812.

Grimm, Wilhelm. „Kinderwesen und Kindersitten“. Kleinere Schriften von Wilhelm Grimm. Band 1. Hrsg. Gustav Hinrichs. Berlin 1881. 359-398.

Kubin, Wolfgang. „Chinas Suche nach einer modernen Literatur“. Moderne chinesische Literatur. Hrsg. Wolfgang Kubin. Frankfurt am Main 1985. 9-17.

Leutner, Mechthild. „,Chinesische Lehre als Grundlage, westliche Lehre zum praktischen Gebrauch': Der Westen aus der Sicht Chinas am Beispiel der Rezeption der YangwuBewegung“. Internationales Asienforum 21.1-2 (1990): 55-70.

Petzoldt, Leander. „Zur Geschichte der Erzählforschung in Österreich“. Homo Narrans. Studien zur populären Erzählkultur. Festschrift für Siegfried Neumann zum 65. Geburtstag. Hrsg. Christoph Schmitt. Münster et. al. 1999. 111-138.

Schede, Hans-Georg. Die Brüder Grimm. München 2004.

Scherer, Anke. Rezension zu „David P. Barrett und Larry N. Shyu (Hrsg.). Chinese Collaboration with Japan, 1932-1945. The limits of Accommodation. Stanford 2001“. Japanstudien 13 (2001): 475-479.

Steig, Reinhold, und Herman Grimm (Hrsg.). Achim von Arnim und die ihm nahe standen. 3. Band: Achim von Arnim und Jacob und Wilhelm Grimm. Stuttgart und Berlin 1904.

Zhou, Zuoren. „Tonghua luelun [Über die Märchen]“. Zhou Zuoren lun ertongwenxue [Zhou Zuoren über die Kinderliteratur]. Hrsg. Liu Xuyuan. Beijing 2014. 25-31. 